\title{
CRITERIOS DE REVISORÍA FISCAL EN LA EVALUACIÓN DE CONTROL INTERNO PARA PREVENCIÓN Y CONTROL DEL FRAUDE
}

\author{
Gregoria Polo-De Lobatón \\ Daulis Lobatón-Polo \\ Sandra Milena Aguirre-Redondo \\ Sacmires Villero-Jiménez
}


Panorama Económico, 24 (Octubre 2016 - Septiembre 2017), pp. 205-220

Gregoria Polo-De Lobatón

Daulis Lobatón-Polo

Sandra Milena Aguirre-Redondo

Sacmires Villero-Jiménez

\title{
Criterios de revisoría fiscal en la evaluación de control interno para prevención y control del fraude
}

\section{Resumen}

La figura del Revisor Fiscal aborda realidades problemáticas en situaciones de fraudes organizacionales, y en muy pocas oportunidades solución a problemas de evaluación del control interno para la prevención y control del fraude. Por lo que la presente investigación, tuvo como pretensión identificar los criterios de Revisoría Fiscal útiles en la evaluación del Control Interno para la prevención y control del Fraude, tomando el caso de una empresa, con pocos resultados en la administración del riesgo, por falta de aplicación de mecanismos adecuados, o falta de una estructura en sus procesos administrativos que ayuden la prevención del riesgo y el fraude. La metodología utilizada con un enfoque cualitativo, de carácter descriptivo, estudio de caso, muestreo caso-tipo, utilizando fuente información primaria en la organización contexto de estudio, y secundaria a partir del análisis documental, utilizando técnicas de entrevistas semi-estructurada y revisión documental. Los resultados de la investigación determinaron que el Control Interno es una garantía para la empresa, con mínimas posibilidades de fallas en la información financiera y garantías del mismo en el cumplimiento; un error en la comprensión de los criterios de Revisoría de Revisoría Fiscal induce al error de juicio y fallas de control; infiriendo que la función del Revisor Fiscal en la fiscalización integral y permanente, con el uso de criterios de Revisoría Fiscal para la evaluación del control interno bajo un enfoque de meta control organizacional, ayuda a la prevención y control del fraude.

Palabras Clave: Revisoría Fiscal, Criterios de Revisorías Fiscal, Control Interno fraude.

Clasificación JEL: M40, M41, M42, M48, M49.

\section{Statutory inspection criteria for the evaluation of internal control to prevent and control fraud}

\begin{abstract}
The figure of the Statutory Auditor entails problematic realities in situations of organizational fraud, and in very few opportunities to solve problems of evaluation of internal control for preventing and controlling fraud. This article is aimed at identifying Statutory Inspection criteria needed in evaluation of Internal Control for preventing and controlling fraud. To do so, we took the case of a company, with few results in risk management, due to lack of implementation of adequate mechanisms, and lack of a structure in their administrative processes for risk and fraud prevention. We used a methodology with a qualitative and descriptive approach, a case study, case-type sampling, primary information sources, and secondary information source from the documentary analysis, using techniques of semi-structured interviews and documentary review. The results of the research determined that the Internal Control is a guarantee for the company, with minimal possibilities of failures in financial information and guarantees for its fulfillment; and that an error, in the understanding of the Statutory Inspection criteria, can lead to error of judgment and control failures. That is why, the Statutory Auditor, in permanent and comprehensive inspection, should help prevent and control fraud, while using Statutory Inspection criteria for the evaluation of the internal control, based on an approach of organizational control.

Keywords: Statutory Inspection, internal control, Statutory Inspection criteria, fraud.
\end{abstract}

JEL Classification: M40, M41, M42, M48, M49.

\section{Critères de révision fiscale dans l'évaluation de contrôle interne pour la prévention et le contrôle de la fraude}

\section{Résumé}

Le rôle du Commissaire aux comptes fait faces à des problématiques lors de la fraude organisationnelle et ne parvient que rarement à résoudre les problèmes d'évaluation du contrôle interne en matière de prévention et de lutte contre la fraude. Dans cet article, nous identifions les critères d'inspection légale nécessaires pour l'évaluation du contrôle interne de prévention et de contrôle de la fraude. Pour ce faire, nous avons pris le cas d'une entreprise qui offre peu de résultats en matière de gestion des risques, manque de mise en place de mécanismes adéquats et souffre de l'absence de structure dans les processus administratifs de prévention des risques et de fraude. Nous avons vu diverses méthodes : qualitative et descriptive, étude de cas, échantillonnage de cas, et source d'information primaire. En organisant le contexte de l'étude comme source primaire et comme source d'information secondaire l'analyse documentaire, des techniques d'entrevues semi-structurées et d'examen documentaire. Les résultats de cette recherche ont déterminé que le Contrôle Interne est une garantie pour l'entreprise, avec des possibilités statistiquement minimes d'échec dans l'information financière et des garanties pour son accomplissement. Une erreur, dans la compréhension des critères d'inspection légale, peut entraîner des aberrations et des défaillances de contrôle. C'est pourquoi le Commissaire aux comptes, en contrôle permanent et complet, devrait contribuer à prévenir et à contrôler la fraude avec les critères de Révision Fiscale pour l'évaluation du contrôle interne, sur la base d'une approche de contrôle organisationnel.

Mots-clés: Révision fiscale, contrôle interne, critères de Révision Fiscale, fraude.

Nomenclature JEL: M40, M41, M42, M48, M49. 


\section{CRITERIOS DE REVISORÍA FISCAL EN LA EVALUACIÓN DE CONTROL INTERNO PARA PREVENCIÓN Y CONTROL DEL FRAUDE}

INFORMACIÓN DEL ARTÍCULO

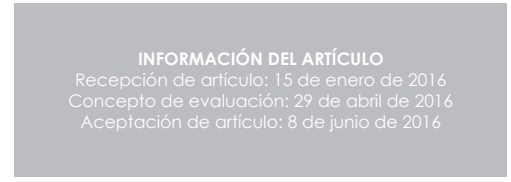

\author{
Gregoria Polo-De Lobatón \\ Universidad Cooperativa de Colombia \\ Colombia \\ Daulis Lobatón-Polo \\ Universidad Cooperativa de Colombia \\ Colombia \\ Sandra Milena Aguirre-Redondo \\ Universidad Cooperativa de Colombia \\ Colombia \\ Sacmires Villero-Jiménez \\ Universidad Cooperativa de Colombia \\ Colombia
}

\section{INTRODUCCIÓN}

Con la presente investigación, se identificó importancia en la aplicación de criterios para la evaluación del Control Interno por parte de la Revisoría Fiscal en la prevención y control del Fraude, se expresa la realidad problemática que abordan los Revisores Fiscales en Colombia ante situaciones de fraudes organizacionales, como los actos que tuvieron lugar por dichos profesionales para prevenir y controlar los riesgos del fraude.

En la actualidad, en algunos Revisores Fiscales es notorio el incumplimiento de actividades relacionadas con el rol que les ha sido asignado socialmente como dinamizadores de la Revisoría Fiscal, entre ellos el control de fiscalización de los entes económicos y la salvaguarda del interés público. Continuamente se vienen registrando en Colombia casos de empresas involucradas en procesos de quiebra, evasión de impuestos, lavado de activos, malversación de fondos públicos y otros tipos de fraudes. En múltiples ocasiones se trató de empresas cobijadas bajo la obligación de contratar la figura de la Revisoría Fiscal y el cuestionamiento a nivel social sobre el papel que cumplieron los Revisores Fiscales que se encontraban allí para el examen de los estados financieros, la evaluación de la transparencia e idoneidad de las actuaciones administrativas, la verificación de la eficiencia y eficacia de las operaciones, la protección de los bienes sociales y, en general, para minimizar los riesgos de la organización y contribuir a asegurar el cumplimiento de sus objetivos.

${ }^{1}$ Autor para correspondencia. Correo electrónico: gregpolo1@hotmail.com; gregopria.polo@campusucc.edu.co 
De continuar con los mismos comportamientos, y en caso de no tomar medidas para contrarrestar la crisis, el fenómeno de pérdida de credibilidad continuará, siendo este uno de los problemas a los que se somete la Revisoría Fiscal, abriendo el espacio a quienes desde todos los ámbitos vienen reclamando su desaparición para sustituirla por la Auditoria Financiera. En efecto, algunos de los protagonistas del caso que se toma como modelo catalogaron la Revisoría Fiscal como prescindible. En consecuencia, se requiere idear propuestas que contribuyan a fortalecerla como institución de fiscalización y control, es en esa vía que se adscribe la presente investigación al apuntar a resolver el siguiente interrogante:

¿Cuáles son los criterios claves a aplicar en la Revisoría Fiscal para la evaluación del Control Interno en la prevención y control del fraude? la finalidad de la presente investigación se propuso identificar $y$ fundamentar criterios claves a aplicar en la Revisoría Fiscal para la evaluación del Control Interno en la prevención del Fraude.

El desarrollo de la investigación se apoyó en los sustentos conceptuales de referencias en autores relacionados con la Revisoría Fiscal, entre ellos a (González G., 2011), Peña, 1998, 2000, 2003; Quintero \& Peñafiel. 2010); en el tema del Fraude a (Cano, 2011), Comité Técnico Local Grupo Guadalajara - Instituto Mexicano de Ejecutivos de Finanzas. (2011), (Estupiñàn, 2006), (Ghirardotti \& Paladini. 2011), (Mejía (2011)), y en el Control Interno a (Mejía H., (2011), (Coopers \& Librand. 1997), (González, 2008), (Llorens \& Fuentes, 2012), (Perdomo,(2004), (Rodríguez , 2010); utilizando en ellos un conceptos que tratan de la intervención de la Revisoría Fiscal en procesos de prevención y control del fraude, estos mismos, sirvieron de apoyo en la construcción de instrumentos para recabar la información.

Por último se presentan los resultados obtenidos a partir de los análisis de ellos, identificando los criterios de Revisora Fiscal útiles para la evaluación del control interno en la perspectiva de la prevención del fraude, con las derivaciones de lineamientos iniciales útiles en el ejercicio de la Revisoría Fiscal con la aplicación de estos.

\section{MATERIALES Y MÉTODOS}

La presente investigación fue de carácter descriptivo con diseño narrativo, reconocida como una forma de recolectar información, que permite describir las categorías objeto de estudio, las relaciones entre ellas, en este caso, la realidad con las características de la categoría criterios de Revisoría Fiscal, en una situación específica, para la evaluación del control interno y los fenómenos asociados a la población bajo estudio para la prevención del fraude, en atención a lo expuesto por (Eyssautier, 2006, p.124) y con rasgos característicos del método Estudio de Caso atendiendo las orientaciones de (Yin, (1998) y (Villareal \& Landeta, 2010).

El diseño de la investigación se orientó por el método estudio de caso, en palabras de (Yin, 1989), consiste en una investigación empírica que investiga un fenómeno contemporáneo en su contexto real, donde los límites entre el fenómeno y el contexto no se muestran de forma precisa, y en el que múltiples fuentes de evidencia son usadas. Pone el énfasis en el objetivo de la investigación, ya que en función de éste se puede considerar que el método se ajusta correctamente cuando 
persigue la ilustración, representación, expansión o generalización de un marco teórico (generalización analítica), y no la mera enumeración de frecuencias de una muestra o grupo de sujetos como en las encuestas y en los experimentos (generalización estadística).

Mencionando a (Villareal \& Landeta, 2010), la cuestión de la generalización de los estudios cualitativos (incluido, el estudio de casos) no radica en una muestra probabilística extraída de una población a la que se pueda extender los resultados, sino en el desarrollo de una teoría que puede ser transferida a otros casos, los mismos autores refiriéndose a (Yin, 1989, 1993, 1994, 1998) y a (Bonache, 1999), los cuales señalan que este tipo de metodología puede servir para describir fenómenos dentro de organizaciones reales, para explorar una situación sobre la que no existe un marco teórico bien definido o para explicar porque se produce un fenómeno como base para la generación de nuevas teorías o para ilustrar buenas prácticas de actuación o validar propuestas teóricas, para nuestro interés los criterios de revisoría Fiscal en la evaluación del control interno para la prevención del fraude.

Los dos autores anteriores, dicen que el estudio de caso es una de los métodos apropiados para aprehender la realidad de una situación estratégica para estudiar un fenómeno en las organizaciones empresariales, para las explicaciones de las relaciones causales complejas, analizar procesos longitudinales, realizar descripciones de perfil detallado, generar teorías o acercar posturas teóricas, tanto de forma exploratoria como explicativa, emplear una perspectiva holística e integral del fenómeno estudiado, entender el contexto real en el que desarrolla el fenómeno analizado en términos generales estudiar un fenómeno sea complejo, ambiguo e incierto.

La fuentes de información esgrimida en la investigación tuvo en cuenta, la primaria en una institución que por su situación legal, estructura, organización y objetivos, además en personas que por sus conocimientos y experiencias, pudieron proporcionar materiales teóricos, empíricos o históricos para llevar a cabo los propósitos de investigación, de igual forma en otras fuentes secundarias en centros de información y documentación, biblioteca y hemeroteca, atendiendo lo dicho por (Rojas, 1989, p.147).

Para recopilar información, se valió de técnicas como entrevistas, revisión documental y observación, con la intención de describir el fenómeno o problemas que resulta de la relación de las categorías objetos de estudio. Aplicando encuesta dirigida al Revisor Fiscal de la empresa con domicilio en Santa Marta como informante clave, y otros empleados, con la intención de dar a conocer los fundamentos teóricos de los criterios claves a aplicar por los Revisores Fiscales en la evaluación del Control interno para la prevención del Fraude, (Rojas, 1989, p.139).

En la misma forma se usó la revisión documental, comprendió todas las actividades relacionada con la búsqueda de información escrita sobre un tema acotado previamente y sobre los cuales se reunieron y discutieron críticamente, toda la información recuperada y utilizada con la intención de dar fundamento a los criterios claves de Revisoría Fiscal para aplicar en la evaluación del Control interno, necesarios en la prevención del Fraude, de acuerdo a (Icart, Fuentelsaz \& Pulpón, 2006, p.12). 
Por las mismas orientaciones el uso de la observación directa para describir los estados, permitió palpar el contexto para describir a forma de narraciones y mapas en notas de campo con anotaciones temáticas descriptivas e interpretativas de los hechos relativos a los procesos que identificaron puntos de control o la falta de estos, (Hernández et, al, 2010).

Elmuestreo:enelcasodelasinvestigaciones cualitativas el tamaño de la muestra no es importante desde una perspectiva probalística es decir solo la cantidad y la estandarización. El objetivo de este estudio se circunscribió en la riqueza, profundidad y calidad de la información, para este estudio se seleccionó como muestreo el Caso-tipo, típica o intensiva, consideró representativa la empresa seleccionada como segmento de la población, de acuerdo a (Hernández et al, 2010).

La validez de manera constructiva, interna y externa para la fiabilidad y consistencia teórico-interpretativa y contextual, utilizando la triangulación de métodos con querencia de múltiples fuentes, entre ellas evidencia documental, observación directa, artefactos físicos, tecnológicos y culturales y la entrevista.

\section{RESULTADOS DE LA INVESTIGACIÓN}

\section{Descripción del Caso de Fraude en la empresa objeto de estudio}

El método diseñado por la investigación, admitió el uso de técnicas de investigación que permitieron detectar situaciones de falta de control interno que guiaron la ocurrencia de fraude. La responsabilidad por la información contable es asignada con criterios confianza por los vínculos de familiaridad entre la gerencia $y$ el departamento de contabilidad, responsabilidades de tesorería ordenada a este departamento, realización de asientos contables sin los soportes, entrega inoportuna en la presentación de los informes contables aduciendo falta de herramientas tecnológicas, inadecuada segregación de funciones, los cuales indujeron a la violación de principio de contabilidad como a normas y principios de auditoria como una falta de un sistema de control interno.

Lo anterior deja percibir un caso de violación a los principios básicos para el ejercicio de la Revisoría Fiscal en el postulado de la confianza pública como define (Peña, 2003), entre ellos independencia, idoneidad, oportunidad y evidencia; falta de un sistema de control interno contable y organizacional en el pensamiento de (Sánchez, 2006), quien señala que el sistema de control interno es uno solo, su desagregación obedece a las exigencias y riesgos que se generan por la existencia de una unidad económica o empresa, cual es la recopilación, clasificación, procesamiento e información de las transacciones contables que representan los hechos financieros, económicos y sociales, productos del desarrollo del objeto social. p. 53.

\section{Criterios de Revisoría fiscal orientadores para la Evaluación del Control Interno bajo la perspectiva de la prevención del fraude}

Criterios presididos por Normas legales de Revisoría Fiscal

a. Normas Internaciones de Auditoría (NIAS)

-NIA 240. Responsabilidad del Auditor al considerar el Fraude y Error en una Auditoría de Estados Financieros. 
Esta Norma Internacional de Auditoría (NIA) establece normas y proporciona lineamientos sobre la responsabilidad del Contador Público en funciones de auditor, considerar el fraude y error en una auditoría de estados financieros. Si bien esta NIA se centra en las responsabilidades del auditor con respecto al fraude y al error, la responsabilidad primaria para la prevención y detección de fraude y error compete en las mismas circunstancias, a los encargados de la administración en una organización.

Son entre otras responsabilidades del auditor, el escepticismo profesional advierte de la discusiòn de la planeación; investigaciones con la administración para la comprensión de la evaluación de la administración del riesgo asegurando que los Estados Financieros presentados en forma errónea representan el resultado del fraude, obtener conocimiento de la comprensión del sistema de contabilidad y del control interno, esclarecer el conocimiento de la investigación del fraude por parte de administración con la identificación de errores de importancia.

De igual forma la norma determina que si una representación errónea identificada puede ser indicativa de fraude, y en su existencia considerar los efectos de la representación errónea con relación a otros aspectos de la auditoría, particularmente la confiabilidad de representaciones de la administración, el cual debe ser documentado ante la presencia de factores de riesgo con todas las implicaciones del proceso.

También, el auditor debe tener conocimiento por escrito de las representaciones de la administración, en los casos de reconocimiento de su responsabilidad en la puesta en marcha y operación de los sistemas de contabilidad y de control interno diseñados para prevenir y detectar fraude y error, por último esta norma reconoce la responsabilidad en la comunicaciòn por parte del auditor cuando identifica una representación errónea resultante de fraude, o un presunto fraude y/o error, o las debilidades de importancia relativa en el control interno, dirigida a la administración, a los encargados del mando y, en algunas circunstancias, a las autoridades de vigilancia y control de la entidad.

\section{-NIA 400. Evaluaciones del Riesgo y Control Interno.}

Esta otra Norma Internacional de Auditoría indica los lineamientos para obtener una comprensión de los sistemas de contabilidad y de control interno sobre el riesgo de auditoría y sus componentes: riesgo inherente a nivel de Estados Financieros, riesgo de control para prevenir o detectar $\mathrm{y}$ corregir representaciones erróneas de importancia relativa y riesgo de detección. $\mathrm{El}$ auditor en su juicio profesional atiende a la evaluación del riesgo de auditoría y al diseño de los procedimientos de auditoría para asegurar que el riesgo se reduce a un nivel aceptablemente bajo.

\section{-NIA 401. Auditoría en un Ambiente de Sistemas de Información Computarizado.}

La Norma Internacional de Auditoría (NIA), hace referencia a los lineamientos relacionados con los procedimientos que debe seguir el auditor cuando conduce la auditoría en un ambiente de sistemas de información computarizado (SIC) Para fines de las NIAs, un ambiente SIC, existe cuando está involucrada una computadora de cualquier tipo o tamaño en el procesamiento de información financiera 
de importancia para la auditoría, sea que esta esté operada por la entidad o por un tercero.

La norma invita al auditor tener las competencias y habilidades especializadas en SIC, para obtener una suficiente comprensión de los sistemas de contabilidad y de control interno afectados por el ambiente SIC y determina el efecto del ambiente SIC sobre la evaluación del riesgo global y del riesgo al nivel de saldo de cuenta y de clase de transacciones, diseñando y desempeñando pruebas de control y procedimientos sustantivos apropiados.

\section{-NIA 500. Evidencia De Auditoría.}

También esta Norma Internacional de auditoría (NIA) establece indicaciones sobre la cantidad y calidad de evidencia de auditoría que se tiene que obtener cuando se auditan estados financieros, y los procedimientos para obtener dicha evidencia de auditoría. El auditor deberá obtener evidencia suficiente apropiada de auditoría para poder extraer conclusiones razonables sobre las cuales basar la opinión de auditoría, utilizando procedimientos de inspección, observación, investigación y confirmación, procedimientos de cómputo y analíticos.

\section{-NIA 520. Procedimientos Analíticos.}

Invita la Norma Internacional de Auditoría (NIA) a la aplicación de procedimientos analíticos durante una auditoría, en las etapas de planeación y de revisión global de la auditoría, simbolizan el análisis de índices y tendencias significativos incluyendo la investigación resultante de fluctuaciones y relaciones que sean inconsistentes con otra información relevante o que se desvían de las cantidades pronosticadas.
Declaraciones Internacionales de Auditoría 1008. Evaluación del Riesgo y el Control Interno - Características y consideraciones del CIS.

Definida de igual manera en la Norma Internacional de Auditoría (NIA) 401, evaluando los controles generales de las aplicaciones de CIS.

Criterios bajo el Pronunciamiento 4Normas Internacionales de Auditoría del C.T.C.P.

Las normas de auditoría generalmente aceptadas (NAGA), han sido los principios fundamentales de auditoría, emplazados para el desempeño de los auditores durante el proceso de la auditoria, garantizan la calidad del trabajo profesional del auditor, se toman como criterios a aplicar por el Revisor Fiscal en la evaluación del Control Interno para la Prevención del Fraude, enmarcados en las normas generales o personales señalando la conducta funcional del auditor como persona humana y regula los requisitos y aptitudes que debe reunir para actuar como Auditor, contemplados también en los Códigos de Ética de otras profesiones, asociados a:

- Entrenamientoycapacidad profesional y Cuidado o esmero profesional.

- Ejecución del Trabajo, regulan la forma del trabajo del auditor durante el desarrollo de la auditoría en sus diferentes fases (planeamiento trabajo de campo y elaboración del informe), orientan a que el auditor obtenga la evidencia suficiente en sus papeles de trabajo para apoyar su opinión sobre la confiabilidad de los estados financieros.

- Planeamiento y Supervisión. 
-Estudio y Evaluación del Control Interno. Como puede verse existe similitud entre las NAGA y las NIAs, es decir el Consejo Técnico de la Contaduría Pública en Colombia, orienta los criterios de Revisoría Fiscal para la evaluación del Control Interno en la perspectiva de la prevención del fraude.

\section{a. Criterios bajo Normas Nacionales}

\section{- Código de Comercio en Colombia, Art. 207.}

Determina las funciones del Revisor Fiscal, y establece entre ellas: a). "Dar oportuna cuenta, por escrito, a la asamblea o junta de socios, a la junta directiva o al gerente, según los casos, de las irregularidades que ocurran en el funcionamiento de la sociedad y en el desarrollo de sus negocios, b). Inspeccionar asiduamente los bienes de la sociedad y procurar que se tomen oportunamente las medidas de conservación o seguridad de los mismos $\mathrm{y}$ de los que ella tenga en custodia a cualquier otro título, c). Impartir las instrucciones, practicar las inspecciones y solicitar los informes que sean necesarios para establecer un control permanente sobre los valores sociales".

\section{-Orientación Profesional del Consejo Técnico de la Contaduría Pública (CTCP).}

La Revisoría Fiscal se rige en su ejercicio, en el campo de control de fiscalización, razón por la cual, son consustanciales con su desarrollo los principios de permanencia, integralidad, oportunidad, función preventiva, independencia y objetividad, fe pública, actuación racional, cobertura general y finalmente, el cumplimiento de las normas de la profesión contable. (p.19), bajo los principios de Permanencia e Integralidad.

\section{-Decreto 302 de 2015}

El decreto en mención, Expide el Marco Técnico normativo de las Normas de Aseguramiento de la información (NAI), que contiene: las Normas internacionales de Auditoria(NlA), las Normas Internacionales de Control de Calidad (NICC); las Normas Internacionales de Trabajos de Revisión (NITR); las Normas Internacionales de Trabajos para Atestiguar (ISAE por sus siglas en inglés); las Normas Internacionales de Servicios Relacionados (NISR) y el Código de Ética para Profesionales de la Contaduría y obliga su aplicación a revisores fiscales de que presten servicios a entidades pertenecientes al Grupo 1 (según decreto 2784 de 2012), y las entidades del Grupo 2 (según decreto 3022 de 2013) que tengan más de treinta mil (30.000) salarios mínimos mensuales legales vigentes (SMMLV) de activo o más de doscientos (200) trabajadores, además de entidades que de manera voluntaria aplicaron el marco normativo de las pertenecientes al grupo 1 .

Estas normas emitidas por el consejo de normas de auditoria y aseguramiento IAASB por su sigla en inglés, están resumidas en el handbook de 2010 de la federación internacional de contadores IFAC por su sigla en inglés, y en ellas se identifica una intención clara de realizar la práctica de la auditoría desde la evaluación y medición del riesgo cuando en sus literales a), b) y c) de la sección 100 párrafo 2 , se enfatiza en las amenazas, indicando tres acciones: identificarlas, evaluar la importancia y aplicar salvaguardas de ser necesario. Lo anterior graficado en la siguiente figura: 
Figura 1. práctica de la auditoría desde la evaluación y medición del riesgo

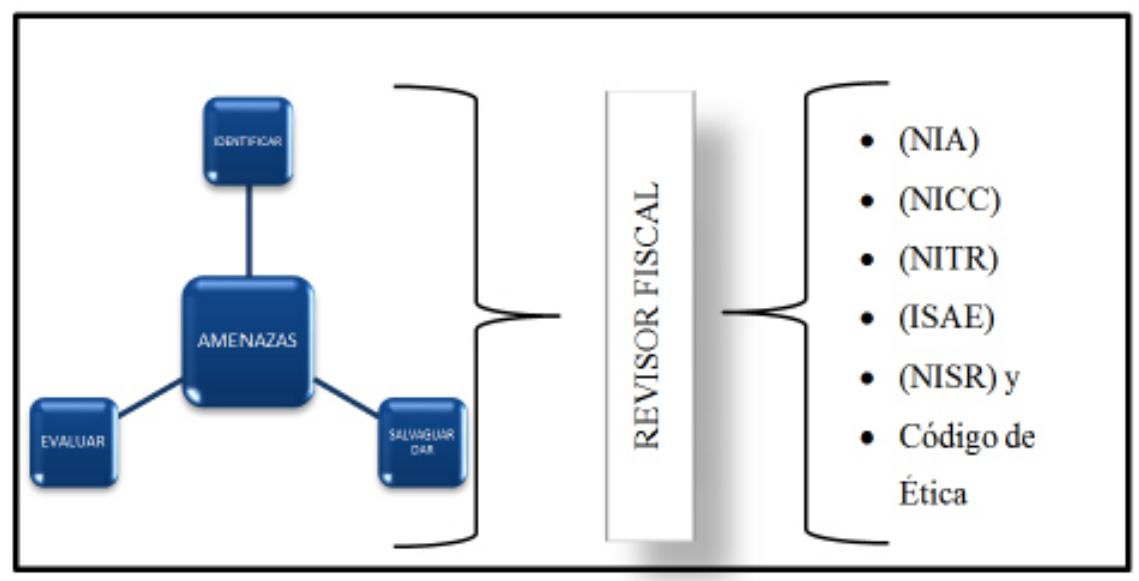

Fuente: adaptación de los autores (2015)

3. Principios como criterios teóricos desde la perspectiva del control y prevención del Fraude.

Algunos autores dedicados a los estudios relacionados con la Auditoría, Revisoría Fiscal y control internos, sirvieron de referentes para indicar de igual manera los criterios de Revisoría Fiscal aplicables en la evaluación del control interno en la perspectiva de la prevención del fraude, entre ellos se seleccionaron a:

-Peña Bermúdez, Jesús María, (1998 y 2003) en los principios del postulado de la confianza pública, La oportunidad, como sinónimo de ocasión, momento, ocurrencia, situación, conveniente, congruente, conlleva a la acción del ejercicio en tiempo real dando valor agregado a la fiscalización, con la acción correctiva hábil, apta, en procura del cumplimiento normativo, previniéndose su violación, señalando los riesgos, causas y consecuencias prevención del fraude. (p.61), Permanencia, referida a la responsabilidad y obligaciones que se derivan de sus funciones y no a la presencia física durante 24 horas-día, con las obligaciones y responsabilidades para obrar con la debida diligencia, (p.65).

-Quintero \&Peñafiel, este autor refiere los elementos para la Prevencion y Control ante el Fraude, a partir de la Protección de la Información y atribuye al Revisor Fiscal velar por la clasificación y custodia, reseñando al Còdigo de Comercio en su Art. 207, bajo la funcion de Inspeccionar asiduamente por los bienes de la sociedad y procurar que se tomen oportunamente las medidas de conservación o seguridad de los mismos y de los que ella tenga en custodia a cualquier otro título. ( p.8); Revelación apropiada, ayudaà a la administración en la protección de los activos de la organización; Controles contables invocando art. 207 numeral 6 del Código de Comercio y Supervisión y control de la gestión, verificando el cumplimiento por parte de la administración, por la existencia de manuales contables apropiados, correcta segregación de funciones, formalización de procedimientos de autorización, supervi- 
sión, registro, custodia y elaboración de la información contable.(p.8)

-Gonzalez, Raul. 2011, se refiere a la revisiòn del Sistema de Control Interno con el uso de técnicas de evaluación como las matrices de riesgo, diagnósticos empresariales, diagramas para evaluación de procesos y cuestionarios de control, que le brindan al Revisor Fiscal una visión detallada del funcionamiento, evaluación y seguimiento de dicho sistema, ( p.8)

-Ghirardotti \& Paladini, atribuye a la evaluación permanente del control interno, una estrategia para mitiguar posibles fraudes; mejora continua del ambiente de control, relacionando de manera directa los planes proactivos de detección de fraudes. (p.18)

\section{Criterios de Revisoría Fiscal aplicados para la Evaluación del Control Interno bajo la Perspectiva de la Prevención del Fraude en un caso empresarial}

Los resultados de la investigación determinan el cuestionamiento en la aplicación de criterios de Revisoría Fiscal para la evaluación del Control interno en la perspectiva de la Prevención del Fraude, en un caso empresarial como objeto de estudio. Los instrumentos utilizados para la obtención de los datos se fundamentaron, en los criterios orientadores resumidos en el numeral 3 de este escrito, con los siguientes resultados:

-Omisión de una actividad, área, operación o función dentro de la labor del Revisor Fiscal, contradice lo afirmado por (Peña, 1998, 2003), respecto a la integralidad refiriéndose a un todo sin excepción alguna a una parte, unidad, operación. Exponiendo la empresa a una mayor probabilidad de riesgo de fraude.
-Las acciones fueron desarrolladas en forma inoportuna en la prevención del fraude,encontraposiciónen lomencionado por Quintero \& Peñafiel acerca de algunas medidas primordiales que debe realizar el Revisor Fiscal en la protección de la información, y por lo cual cita el Código de comercio en su art. 207 "Inspeccionar asiduamente los bienes de la sociedad y procurar que se tomen oportunamente las medidas de conservación o seguridad de los mismos y de los que ella tenga en custodia a cualquier otro título".

-En la misma forma se desconocio los resultados de las operaciones reveladas en los informes de gestiòn, sin dar cuena de la evaluación continua y resultados obtenidos, más recomendaciones necesarias a fin de implementar medidas de control. Como lo recomienda Quintero y Peñafiel como otra medida de prevención y control ante el fraude.

-Falta en la aplicación de esquemas o técnicas de evaluación como los Diagnósticos empresariales, ante la revisión del Sistema de Control Interno como lo ha expresado por Gonzalez.

-Falta existencia de un plan de mejora continua del ambiente de control, relacionando con planes proactivos de detección de fraude. Contradiciendo lo recomendado por Ghirardotti \& Paladini.

-Revelación por la no realización de la respectiva investigación con la administración para obtener conocimiento y comprensión de la administración respecto a los sistemas de contabilidad y de Control Interno y por supuesto la falta de comunicaciòn de cualquier debilidad de importancia relativa, establecida para prevenir y detectar error en el mismo, de acuerdo a lo establecido por la NIA 240. 
-Falta de evaluación en la afectación de los controles generales de las aplicaciones de C.I.S, contradiciendo lo establecido por la Declaración Internacional de Auditoría 1008.

Es importante reconocer que la acción de la Revisoría Fiscal en la empresa presume ser amplia, bajo los supuestos de la función notable depositario de la fe pública, en el significado de la atestación o dictamen del Revisor Fiscal, el cual prevé que el acto se ajusta a los criterios de Revisoría Fiscal establecidos en normas legales, estatutarios, técnicos y previsionales del (C.T.C.P, 2008, p.18). Es por tanto, que su integralidad se enfoca en la revisión de cada parte funcional de la empresa como un todo, para hallar las anomalías que afecten esos requisitos legales, estatutarios, técnicos y previsionales, conjunto al apoyo de los demás profesionales de diferentes campos.

\section{Lineamientos iniciales para la aplicación de criterios de Revisoría Fiscal en la evaluación del Control Interno bajo la perspectiva de la prevención del Fraude.}

La Revisoría Fiscal, es la institución que nace como respuesta de una necesidad social, proporciona confianza pública en la gestión fiscalizadora del ente económico, garantiza un enfoque de controles al minimizar los riesgos; en el mismo sentido la organización cumple el régimen normativo en la viabilidad empresarial, asegurando en los Estados Financieros un reflejo de la realidad financiera del sistema empresarial.

Valga aclarar con lo anterior, que las causas del Fraude pueden originarse en donde existan debilidades en las estructuras y controles de las empresas, posibilitando la acumulación de maniobras fraudulentas, esto porque ocurre con mayor frecuencia en pequeñas y medianas empresas, por inadecuados controles internos 0 inexistencia de los mismos, señalando la confianza más en las personas que en los procesos, y la falta de ella por la dirección. (Quintero \& Peñafield, 2010, p12).

En las mismas orientaiones, los procedimientos utilizados por el revisor fiscal para reducir el fraude en la empresa se relacionan en forma directa con los principios de auditoría generalmente aceptados que deben ser realizados en forma permanente por el profesional cubriendo todas las áreas de la organización, especialmente aquellas identificadas como susceptibles de fraude, como por ejemplo el conjunto de técnicas de investigación aplicables a una partida o a un grupo de hechos o circunstancias relativas a los estados financieros, (Quiñonez, 2008).

Sin dejar por fuera los elementos de control interno para la prevenciòn del fraude entre ellos, protección de la información, revelación apropiada, controles contables, supervisión y control de la gestión, realizar una evaluación independiente, para determinar que tanto las normas, políticas y procedimientos estipulados por la compañía se estén cumpliendo por parte de la administración, veirficando la existencia de manuales contables apropiados, adecuada segregación de funciones, formalización de procedimientos de autorización, supervisión, registro, custodia y elaboración de la información contable. (Quintero y Peñafiel. 2010).

En la revisión del Sistema de Control Interno se utilizan y aplican esquemas o técnicas de evaluación como las matrices de riesgo, diagnósticos empresariales, diagramas para evaluación de procesos y 
cuestionarios de control, que le brindan al Revisor Fiscal una visión detallada del funcionamiento, evaluación y seguimiento de dicho sistema, verificando la implementación de sus principales componentes como son: Ambiente de control, valoración de riesgos, actividades de control, información y comunicación, monitoreo, el control interno, no cosiste en un proceso secuencial, en donde alguno de los componentes afecta solo al siguiente, sino en un proceso multidireccional repetitivo y permanente, en el cual más de un componente influye en los otros y conforman un sistema integrado que reacciona dinámicamente a las condiciones cambiantes.

Lo anterior determina, que con la aplicación de procedimiento de control, se pueden prever los fraudes, utilizando exámenes crítico, revisión, evaluación de actos, de los procedimientos, documentos, estados financieros, asumiendo niveles de evaluación de control permanente, oportuno e integral, convirtiendo así al control como una actividad permanente dando su enfoque a la Fiscalización, Supervisión y vigilancia en tiempo real de las operaciones de las actividades de la empresa.

Además, al estudiar y evaluar el Control Interno, el Revisor Fiscal diseñará estrategias de desarrollo, con esta información concluirá el fundamento, procedimientos y finalidad, determinando posibles riesgos, excepciones de controles internos, deficiencias e inconsistencias y, algunas veces, indicios de irregularidades. Ellos debe ser analizados bajo los parámetros de importancia relativa y materialidad y hacerlos conocer a los clientes, suministrando recomendaciones y algunas veces diagnósticos para que la administración determine medidas preventivas para que se proyecten los arreglos necesarios dentro de la organización.

El Revisor Fiscal con el uso del juicio profesional para evaluar el riesgo y diseñar los procedimientos para asegura la reducción a un nivel aceptable bajo, en orientaciones de las Normas Internaciones de Auditoria, revisión de controles en la aplicación de CIS. En consecuencia, puede ser más eficiente revisar el diseño de los controles generales antes de revisar los controles de aplicación.

\section{CONCLUSIONES}

Buscando una reflexión sobre la importancia de la responsabilidad del Revisor Fiscal en la evaluación del sistema de Control Interno, función propia para la intervención en los procesos de prevención y control de fraudes organizacionales, que permita alertar de posibles situaciones sobre el cumplimiento de normas, leyes y estándares internacionales definidos, se puede inferir que:

Se reveló la realidad problemática al describir características contables y administrativas de una empresa objeto de estudio, la cual presentó irregularidades en su estructura de Control Interno, manteniendo una mayor posibilidad a los riesgos de fraude, denotando la importancia que conlleva la continua evaluación del control interno por parte del Revisor Fiscal en los procesos de prevención y control del fraude, atendiendo al cumplimiento de los objetivos establecidos por el código de comercio, CTCP y otras disposiciones normativas como a principios, todos alusivos a las funciones en la Institucionalidad de la Revisoría Fiscal . 
Se identificaron criterios claves a aplicar en la Revisoría Fiscal para la evaluación del control interno bajo la perspectiva de la prevención y control del Fraude, como criterios para la planeación, ejecución del trabajo y divulgación del juicio profesional, fundamentados en Normas internacionales (NIAs), Normas de aceptación general de Auditoría (NAGA) y orientaciones Nacionales en Colombia determinadas por el CTCP, Código de Comercio, como principios aplicados en los criterios de Revisoría Fiscal.

Se detectó que en un caso de una empresa objeto de estudio, la falta de aplicación de criterios Revisor Fiscal en el momento de evaluar la estructura del Control Interno para prevenir y controlar el fraude. Concluyendo que la falta de aplicación de criterios de Revisoría Fiscal en la evaluación del control interno, el Revisor Fiscal comete errores de juicio en su examen, dando la oportunidad en la comisión del fraude y el error, infiriendo que la función del Revisor Fiscal obedece a una fiscalización integral, permanente, preventiva, e independiente de la administración con enfoque de meta-control organizacional (controla los sistemas de control organizacionales).

\section{REFERENCIAS BIBLIOGRÁFICAS}

Cano, D. (2011). Contra el Fraude. Prevención e Investigación en América Latina. Recuperado de: http://books.google.com. co/books?id=i7T5pszT2eQC\&printsec=fron tcover\&hl=es\#v=onepage\& $\mathrm{q} \& \mathrm{f}=$ false.

Cepeda A. G. (1997). Auditoría y Control Interno. Bogotá: McGraw-Hill INTERAMERICANA, S.A. (P.17)

Comité Técnico Local Grupo Guadalajara - Instituto Mexicano de Ejecutivos de Finanzas. (2011). Detección y Prevención de Fraudes. Recuperado de: http://www.imef. org.mx/grupos/guadalajara/descargas/ fraudes/Fraudes.pdf
Consejo Técnico de la Contaduría Pública. (2008). Orientación Profesional de la Revisoría Fiscal. Recuperado de: http://www. actualicese.com/normatividad/2008/ Otras/OrientacionProfesional/OP-21-062008.pdf

Consejo Técnico de la Contaduría Pública. Pronunciamiento 4 - Normas de Auditoría Generalmente Aceptadas. Recuperado de: http://www.cijuf.org.co/CTCP/ pronunciamientos/PRONUNC4.pdf

Coopers \& Librand. (1997). Nuevos conceptos del control interno. Recuperado de: http:// books.google.es $/$ books?id=335uGf3nusoC\& printsec $=$ frontcover $\& \mathrm{hl}=\mathrm{es} \# \mathrm{v}=$ onepage $\& \mathrm{q}$ $\& \mathrm{f}=$ false

Decreto $\mathrm{N}^{\circ}$ 2784. Diario oficial de la Republica de Colombia, Bogotá D.C, 29 de diciembre de 2012. $\mathrm{N}^{\circ} 48.648$.

Decreto $\mathrm{N}^{\circ}$ 3022. Diario oficial de la Republica de Colombia, Bogotá D.C, 27 de diciembre de 2013. $\mathrm{N}^{\circ} 49.016$.

Decreto $\mathrm{N}^{\circ}$ 0302. Diario oficial de la Republica de Colombia, Bogotá D.C, 20 de febrero de 2015. $\mathrm{N}^{\circ} 48.648$.

Estupiñan Gaitán, R. (2003). Control interno y fraudes con base en los ciclos transaccionales. Análisis de informe COSO 1 y 2. Colección textos universitarios. Recuperado de: http://books.google.com.co/ books?id=8Yvg8u2pD94C\&pg=PA277\&dq= el+auditor+de+operaciones\&hl=es-419\&sa $=X \& e i=8 X c i U e C 5 E o a 08 A S D q I C Y A g \& v e d$ $=0 C F k Q 6 w E w B w \# v=$ onepage $\& \mathrm{q}=\mathrm{el} \% 20$ auditor\%20de\%20operaciones\&f=false

Eyssautier M. (2006). Metodología de la investigación: desarrollo de la inteligencia. Recuperado de : http://books.google.com. co/books?id=xdALJ4BXo_AC\&pg=PA124 $\& d q=$ tipo+de+estudio++investigacion\&hl $=$ es419\&sa $=X \&$ ei $=001 Z U o S r M I z m 8 w S t v 4$ BA\&ved $=0 C C w Q 6 A E w A A \# v=$ onepage\& q=tipo\%20de\%20estudio\%20\%20investigacion\& $\mathrm{f}=$ false

Ghirardotti \& Paladini. (2011). Métodos de prevención, detección e investigación de fraudes dentro de empresas. Recuperado de: http://www.palermo.edu/economicas/ contadores/presentaciones/Binder1.pdf. 
González-Cueto \& Pando. (2006). La administración de Riesgos Empresarial en el contexto actual del control interno. Recuperado de: http://www.nodo50.org/ cubasigloXXI/economia/gcueto_311206. pdf

González Garzón, R. E. (2011). La responsabilidad del revisor fiscal en la evaluación del control interno. Recuperado de: http:// www.javeriana.edu.co/fcea/eventos_rev_ fiscal/III_revisoria_fiscal/ponencia_raul_ gonzalez_u_externado.pdf

González, M. (2008). Influencia de la ética del contador Público en el control Interno de las organizaciones. Recuperado de: http://servicio.bc.uc.edu.ve/faces/revista/ vol19n2/art3.pdf.

Hernández, S., R., Fernández, C., C., Baptista, L., P., (2010), Metodología de la Investigación, Editorial McGraw Hill, Educación, Interamericana Editores, S.A. de C.V. Quinta Edición.

Icart, Fuentelsaz \& Pulpón. (2006). Elaboración y Presentación de un proyecto de Investigación y una Tesina. Recuperado de: http://books.google.com.co/books?id=5CW KWi3woi8C\&pg=PA12\&dq=revision+biblio gr\%C3\%A1fica+es\&hl=es-419\&sa $=X \& e i=x 5$ dbUpKKB4q09QT1kYDgDA\&ved=0CDUQ 6AEwAg

KPMG en Colombia. (2011). Encuesta de Fraude en Colombia 2011. Recuperado de:http://www.kpmg.com/CO/es/ Issues AndInsights/ArticlesPublications/ Documents / Encuesta $\% 20$ de $\% 20$ Fraude\%202011.pdf

Ley 222de1995. CódigodeComercio. Recuperado de: http://www.secretariasenado.gov.co/ senado/basedoc/ley/1995/ley_0222_1995. html

Llorens, F. \& Fuentes, M. (2012). Control. Recuperado de: http://descuadrando.com/ Control.

Mantilla, S.,A .,(2003)Control Interno, Informe COSO, Ediciones Ecoes, Tercera Ediciòn.

Mejía Hernández, M. (2011).La Auditoria en el descubrimiento de Fraudes Financieros. Recuperado de:http://cdigital. uv.mx/bitstream/123456789/32187/1/ mejiahernandezmaria.pdf
Normas internacionales de Auditoría. Recuperado de: http://fccea.unicauca.edu. co/old/nias.htm.

Peña, B., J., M., (1998), El control, La Auditorìa, y la Revisorìa Fisacal. Ediciones Ecoes, Colombia.

Peña Bermúdez, J. M. (2000). Control, Auditoría, y Revisoría Fiscal. Incluye Contraloría y Ética profesional. Recuperado de: http:// books.google.com.co/books?id=AYBhlN6E Lu0C\&pg=PA87\&dq=revisor + fiscal\&hl=en \&sa=X\&ei=LfgkUbHqK4Oi8gTI5YHYBQ\&v ed=0CEEQ6AEwAw.

Peña Bermúdez, J. M. (2003) ¿Interventoría o Auditoría?. Impresiòn litografía Mercurio. Bogotá, D.C, Colombia.

Perdomo Moreno, A. (2004). Fundamentos del Control Interno. Recuperado de: http:// books.google.com.co/books?id=VobCCBs MJtoC\&pg=PA3\&dq=prevenci\%C3\%B3n $+\mathrm{y}+$ control+de+fraude\&hl=es\&sa=X\&ei= uAtZUfGpJKXC4AOY7oCQAQ\&ved=0C C4Q6AEw ADgK\#v=onepage\& $\mathrm{q}=$ preven ci\%C3\%B3n\%20y\%20control\%20de\%20 fraude $\& \mathrm{f}=$ false

Quintero \&Peñafiel. (2010). La acción de la revisoría fiscal frente al fraude en las empresas. Recuperado de: http://repository. unimilitar.edu.co/bitstream/10654/3682/1/ QuinteroCameroLibiaInes2010.pdf

Quiñones, J.(2008).Procedimientos y técnicas de auditoria. Recuperado de : http://www. gerencie.com/procedimientos-y-tecnicasde-auditoria.html.

Rodríguez Medina, H. (2010). Mapa de riesgos. Control interno. Recuperado de: https:// sites.google.com/site/mecicalidad/mapade-riesgos-control-interno.

Rojas Soriano, R. (1989). Investigación social: Teoría y Praxis. Recuperado de: http:// books.google.com.co/books?id=a5A-au7 zn7YC\&pg=PA4\&dq=Rojas + Soriano, $+\mathrm{Ra}$ \%C3\%BAl+(1989).+Investigaci\%C3\%B3n +social+teor\%C $3 \% A D a+y+$ praxis\&hl=es419\&sa=X\&ei=XXhbUrnAJ4_69gSwooHg Dw\&ved=0CEQQ6AEwBA\#v=onepage\& $q=$ Rojas\%20Soriano\%2C\%20Ra\%C3\%BAl\%20 (1989).\%20Investigaci\%C3\%B3n\%20 social $\% 20$ te or $\%$ C $3 \%$ ADa $\% 20$ y $\% 20$ praxis\&f=false. 
Sánchez, Ch., W., (2006), Control Interno, Conceptual y practico. Investigar Editores. Pereira, Colombia.

Villarreal L., O. y Landeta R., J. (2010), El estudio de casos como metodología de investigación científica en dirección y economía de la empresa. Una aplicación a la internacionalización. Universidad del País Vasco, Investigaciones Europeas, Vol. 16, No 3, 2010, pp. 31-52, Mayo. Documento descargado de http://www.elsevier.es el 06/06/2014.

YIN, R. K. (1989): Case Study Research. Design and Methods, Applied Social Research Methods Series, Vol. 5, Sage Publications, London.
YIN, R. K. (1993): Applications of case study research, Sage Publications, London.

YIN, R. K. (1994): Case Study Research. Design and Methods, Applied Social Research Methods Series, Vol. 5, Second Edition, Sage Publications, London.

YIN, R. K. (1998): "The Abridged Version of Case Study Research", en BICKMAN, L. y ROG, D. J. (eds.): Handbook of.

Polo-De Lobatón, G., Lobatón-Polo, D., AguirreRedondo, S., \& Villero-Jiménez, S. (2016). Criterios de revisoría fiscal en la evaluación de control interno para prevención y control del fraude. Panorama Económico, 24, 205-220.

\section{AUTORES}

Gregoria Polo-De Lobatón

Doctora en Ciencias Gerenciales de la Universidad Privada Dr. Rafael Belloso Chacín (Venezuela). Magíster Ciencia en Administración Financiera y de Sistemas de la Universidad Central de Bogotá en convenio con la Universidad del Quindío (Colombia). Especialista en Revisoría Fiscal y Control de Gestión; Especialista en Docencia Universitaria de la Universidad Cooperativa de Colombia (Colombia). Contadora Publica de la Universidad del Quindío (Colombia). En la actualidad Profesor de Tiempo Completo Investigador de la Facultad de Ciencias Administrativas Contables y de Comercio Internacional de la Universidad Cooperativa de Colombia (Colombia). Adicionalmente, conferencista y facilitadora nacional e internacional.

\section{Daulis Lobatón-Polo}

Doctor en Ciencias Gerenciales. Magister en Contabilidad Internacional y Auditoría de Gestión. Especialista en Revisoría Fiscal y Control de Gestión y Gestión Tributaria y Aduanera. Contador Público. Docente de la Universidad Cooperativa de Colombia Sede Santa Marta.

Sandra Milena Aguirre-Redondo

Contador público de la Universidad Cooperativa de Colombia. 